\title{
Efficient Content based Image Retrieval System using Mpeg-7 Features
}

\author{
Swapnalini Pattanaik \\ M.E. Electronics Student, \\ RSCOE,Pune,India.
}

\author{
D.G. Bhalke \\ HOD,EL\&TC, \\ RSCOE,Pune,India
}

\begin{abstract}
This paper gives an overview idea of efficient retrieval of images using different Mpeg-7 Features. Content Based Image Retrieval is a technique of automatic indexing and retrieving of images from a large data base. Feature Extraction and Similarity Matching are the two major steps for CBIR Systems. Color, Texture and Shape represent the three visual features for any image. Mpeg-7 Stands for Multimedia Content Description Interface. The main objective of Mpeg-7 is to provide a standardized set of technologies for describing multimedia content. It has allowed quick and efficient content identification, and addressing a large range of applications. The visual descriptors are classified according to the feature such as color, shape, texture, etc. This paper has used Color Structure Descriptor for color and Edge Histogram Descriptor for texture.These two features are also integrated to increase the performance of CBIR Systems. The efficiency of all methods are demonstrated with the help of results.
\end{abstract}

Keywords - Content Based Image Retrieval, Color Structure Descriptor(CSD), Edge Histogram Descriptor(EHD), MPEG-7

\section{INTRODUCTION}

Due to the rapid advancement in science and technology more and more images are produced every day. So it is very much needed to efficiently store, index ane retrieve images. There are two methods are present for this. The traditional method is the Text based image retrieval.

In Text based Image Retrieval images are indexed by meta data. It is a very easy and simple method for small database. When database is large it is very difficult to index the image by a proper word. it is also so very tedious.

To remove this problem content based image retrieval system has developed. Feature extraction and similarity matching are the footsteps for image retrieval. CBIR has used the visual feature such as color, texture and shape.

The amount of multimedia content available in digital archives, on the World Wide Web, in broadcast data streams and in personal and professional databases is growing out of control. This challenging situation led to the need of a solution to the problem of quickly and efficiently searching for various types of multimedia material interesting to the user. In October 1996, the Moving Pictures Expert Group (MPEG) started a new work item to provide a solution to the above problem. The newest member of the MPEG family, called the multimedia content description interface (MPEG7). The goal of the MPEG-7 standard is to provide a rich set of standardized tools to describe multimedia content. and in particular audio-visual content. The previous MPEG standards (MPEG-1, MPEG-2, MPEG-4), which have mainly addressed coded representation of multimedia content. In MPEG-7 the description tools are applicable for all kinds of multimedia content independent of its format and coding. MPEG-7 aims to standardize a core set of quantitative measures of audiovisual features, called Descriptors (D), and structures of descriptors and their relationships, called Description Schemes (DS) in MPEG-7. MPEG-7 will also standardize a language - the Description Definition Language (DDL) - that specifies Description Schemes to ensure flexibility for wide adoption and a long life. The visual descriptors, which are specified in this standard, define the syntax and the semantic of each feature (meta-data element). These descriptors are classified according to the feature which is described, such as color, shape, texture, etc. MPEG-7 has five color descriptor named as Dominant Color,Scalable Color, Color Structure, Color Layout and Group of Frames/Group of pictures Color.There are two descriptors related to texture known as Homogenous Texture Descriptor and Non-Homogenous Texture Descriptor (Edge Histogram). The Visual Shape Descriptors consists of 3-D Shape Descriptor, Region-Based Descriptor, Contour-Based Shape Descriptor, 2-D/3-D Shape Descriptor. There are four Motion Descriptors named as Motion Activity Descriptors,Camera Motion Descriptors,Motion Trajectory Descriptors, Parametric Motion Descriptors.

My paper has used Color Structure Descriptor and Edge Histogram Descriptor for feature extraction .The Related Work is discussed in Section 2. Section 3 Covers the explanation of Proposed System .The Result and Discussion is reflected in Section 4. The Conclusion of the paper is discussed on Section 5.

\section{METHODOLOGY}

There are various approaches are present for Content Based Image Retrieval. Some of the important literature which covers the more important CBIR System is discussed below.

Chin-Chin Lai et.al. [1] have proposed an interactive genetic algorithm (IGA) to reduce the gap between the retrieval results and the users' expectation .They have used color attributes like the mean value, standard deviation, and image bitmap .They have also used texture features like the entropy based on the gray level co-occurrence matrix and the edge histogram. They compared this methods with others approaches and achieved better results.

Meenakshi Madugunki et.al.[2] have published a paper on detailed classification of CBIR Systems. They have used the Global color histogram, Local Color histogram, HSV method for extracting the color feature and matched the result by using Euclidean distance, Canbera distance and city block distance. They have also used discrete wavelet transform (DWT) for Texture Feature extraction and compared the result obtained by using different features.

C. Vertan et.al.[3] have proposed a generalized structure descriptor (GSD) in the paper. The standard MPEG-7 color structure descriptor (CSD) is a particular case of the proposed GSD. The GSD descriptor embed color and spatial 
distribution information, allowing a compact description of color and texture to be used in content-based image retrieval. The Experimental result showes that the proposed descriptors can be successfully used for color texture and general image recognition and retrieval.

Wu Siqing[4] et.al have introduced the three key aspects i.e. the description to shape feature, the extraction shape feature, shape match to realize image retrieval based on shape feature and also introduced the standard of multimedia-contentdescription interface - MPEG-7.

Jian-hua li et.al. [5] have analyzed the color space used by MPEG-7 Color Layout Descriptor. They have used HSV instead of original color space. They have also compared the result of HSV with HSL and YCbCr.The performance of $\mathrm{HSV}$ is more than the HSL and $\mathrm{YCbCr}$.

Hong Shao et.al.[6] have proposed a new texture feature known as wedge feature in the paper. It has emphasized the texture direction. The homogeneous texture descriptor owning the new feature is more suitable to content-based image retrieval system. The experimental results show this method can get better retrieval recall and accuracy ratio comparing with that of only using the texture energy or the generalized gray co-occurrence matrix.

S. Kiranyaz et.al. [7] have addresed dominant color extraction as a dynamic clustering problem and used techniques based on Particle Swarm Optimization (PSO) for finding optimal (number of) dominant colors in a given color space, distance metric and a proper validity index function. They have also proposed an efficient color distance metric, which uses a fuzzy model for computing color (dis-) similarities over HSV (or HSL) color space. The comparative evaluations against MPEG-7 dominant color descriptor show the superiority of the proposed technique

M. Koyuncu et.al.[8] have proposed two different index structures, namely Slim-Tree and BitMatrix, for efficient retrieval of images based on multidimensional low-level features such as color, texture and shape. These index structures also use metric space. They have used MPEG-7 Descriptors extracted from images to represent these features and store them in a native XML database. color layout (CL), dominant color (DC), edge histogram (EH) and region shape (RS) are used in Slim-Tree and BitMatrix .They are aggregated by ordered weighted averaging (OWA) method to find final similarity between any two objects.

Aleksey Fadeev et.al [9] have approached a generic approach for representing image texture features approach, called Dominant Texture Descriptor (DTD.This method is based on clustering the local texture features and identifying the dominant components and their spatial distribution. They have also used an enhanced version of the DTD (eDTD) that encodes the spatial distribution of the pixels within each dominant component.They have also compared the result with two well-known descriptors,i.e MPEG-7 Edge Histogram, and Gabor texture. The performance of the proposed texture feature has given better result .They have used a database consists of 900 color images.

Ka-Man Wong et.al. [10] have introduced a new mechanisim known as Dominant Color Structure Descriptor (DCSD) for efficient representation of both the color and the spatial structure information with single descriptor.This method has combined the compactness of Dominant Color Descriptor (DCD) and the retrieval accuracy of Color Structure Descriptor (CSD). The new similarity measure algorithm based on matching similar colors to generate a common palette, instead of using a fixed histogram space, is developed for DCSD. Experiment results show that DCSD gives better retrieval performance than the original DCD.

From the literature survey it is concluded that a wide variety of CBIR algorithms have been proposed in different papers. The performance of the system varies from paper to paer depend upon the algorithm used . My paper has used both color structure descriptor and edge histogram descriptor. It has also combined the two features and implement on the system.

\section{SYSTEM IMPLEMENTATION}

The system has used MPEG-7 features to increase the performance. Query image is taken as the input to the system. Then internal processing is done. After that top-k images are displayed on the screen. In MPEG-7 different types of descriptors are present. I have used color structure descriptor and edge descriptor for my process.

Feature Extraction and Similarity matching are the two basic steps for any image retrieval system. Depending upon the selection of feature the output is varied. My system has used four steps for giving output. The detailed description and diagram is given below.

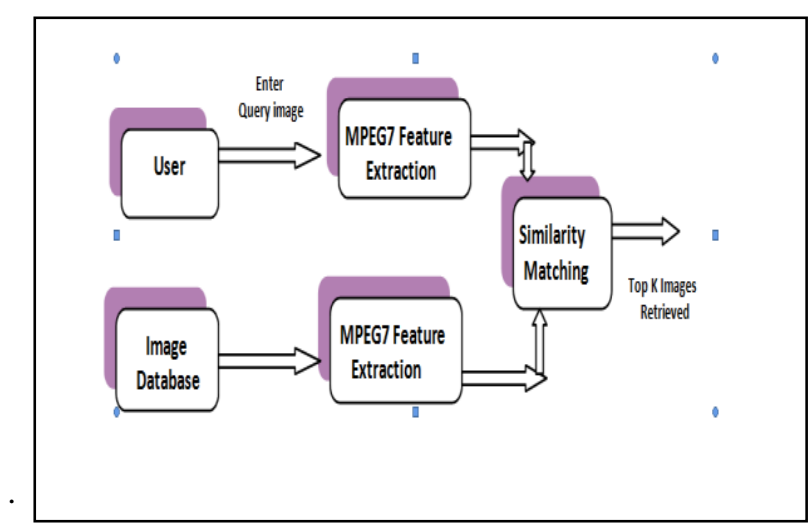

Fig 1.Block Diagram

The proposed system has the following structure

1) Collection of Database: A database containing no of mages with any one of the formats .bmp, .jpg, .tiff. is required.

2) Query: The user provides a sample image or sketched figure as the query for the system.

3) Feature Extraction: There are various kinds of low-level visual features to represent an image, such as color, texture, shape, and spatial relationship.In my paper I have used color structure descriptor and edge histogram descriptor for feature extraction. Detailed about all the features are given below:

\section{Color Structure Descriptor:}

Color structure descriptor is a color histogram in the quantized Hue Max Min Difference (HMMD) color space.It captures both color content and information about the structure of this content.This descriptor can distinguish two images in which a given color is present in same amount but Structure of the group of pixel having that colors are different.So it gives better result than the traditional histogram. The Color Structure Descriptor expresses local color structure in an image using an $8 \mathrm{X} 8$ structuring element 
that scans the image (Raster scanning method), it counts the number of times a particular color is contained within the structuring element. The CSD Extraction steps are given below.

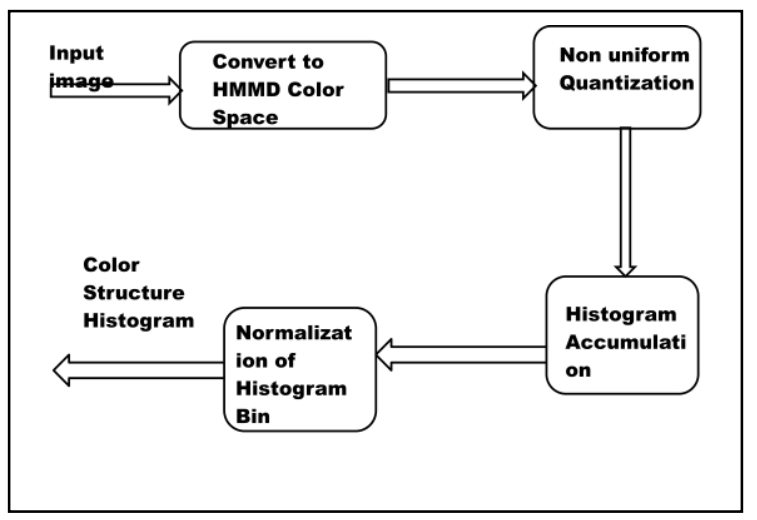

Fig 2.Color Structure Descriptor Block Diagram

The input image of different color space is converted to HMMD Color space. HMMD color space, is supported in MPEG-7. The hue has the same meaning as in the HSV space, and max and min are the maximum and minimum among the $\mathrm{R}, \mathrm{G}$, and $\mathrm{B}$ values, respectively. The diff component is defined as the difference between max and min. The three of the four components are sufficient to describe the HMMD space(Hue, Min, Max) or (Hue, Diff, Sum). This color space is represented by double cone structure. The HMMD color space is very effective and compared favorably with the HSV color space.

HMMD is nonuniformly quantized using 256, 128, 64 or 32 quantization level.

The next step is the histogram generation. It is obtained by passing a $8 * 8$ structuring element throughout the image. The number of bins of the histogram is same as the no of quantization level.For each position, if a quantized color $\mathrm{c}_{\mathrm{n}}$ belongs to the element, the corresponding bin of the histogram is incremented in one unit, independently of the number of pixels which have the same value $c_{n}$. The below diagram represents an example with a quantization of 8 levels.

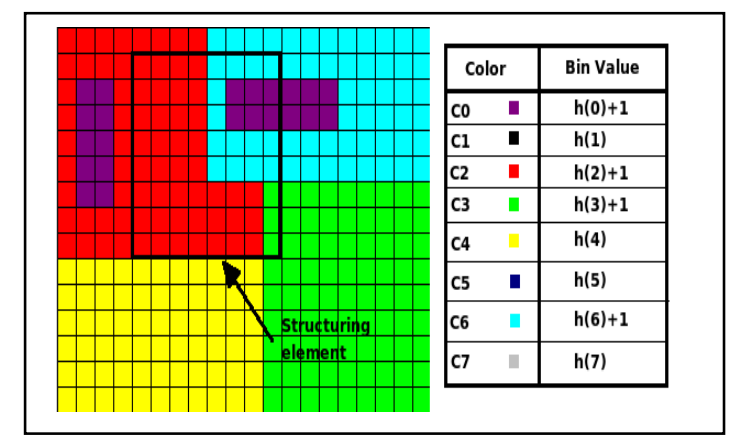

Fig 3.Csd Histogram Generation by passing $8 * 8$ Structuring element
The final step is the Normalization of Histogram and non uniform quantization of each bin amplitude to 8 bit code. The bin amplitudes are normalized by $\mathrm{h}_{\max }$ and $\mathrm{h}_{\max }$ reprents the total location covered by the structuring element within the image.

\section{Edge Histogram Descriptor:}

Edge Histogram is one of the important types of texture descriptor of MPEG-7. Edges in images constitute an important feature to represent their content.

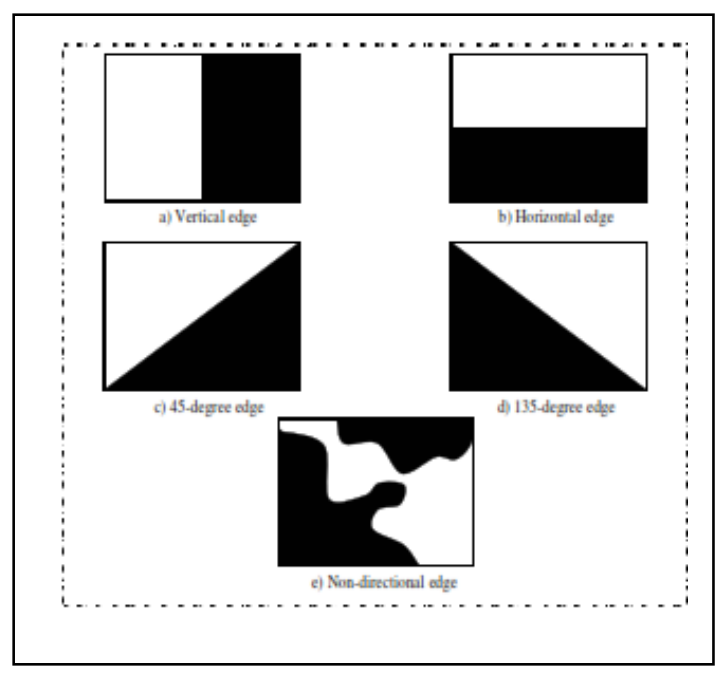

Fig 4. Structure of different types of Edges

Human eyes are sensitive to edge features for image perception. One way of representing such an important edge feature is to use a histogram. An edge histogram in the image space represents the frequency and the directionality of the brightness changes in the image.

The histograms for each block could also represent the occurrence frequencies for 5 types of edge, called as vertical,horizontal, 45-degree diagonal, 135-degree diagonal and non-directional edge bin. The 5 types of histograms are calculated for local block,semi global block,global block.

The local block is generated by dividing the the images into $4 * 4$ sub images.Each subimage is then divided into no of non overlapping blocs depenind upon the size of image.Each block is divided into $2 * 2$ blocks. The diagram is given below.

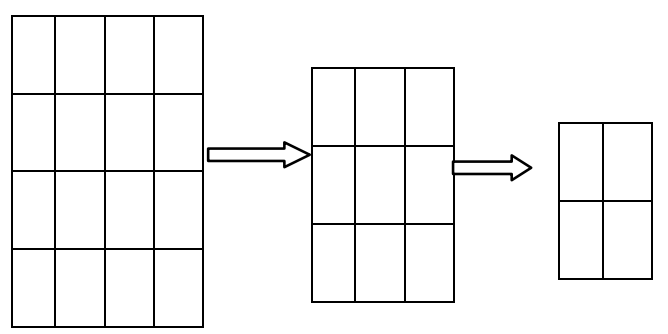

Image Sub Image block

Fig 5.Local block Generation 
For each sub images 5 types of edges are calculated.The histogram of each subimage consists of five no of bins.So $(16 * 5) 80$ bins are generated in local block.13 semigobal blocks are generated by combining 4 consecutive local blocks.

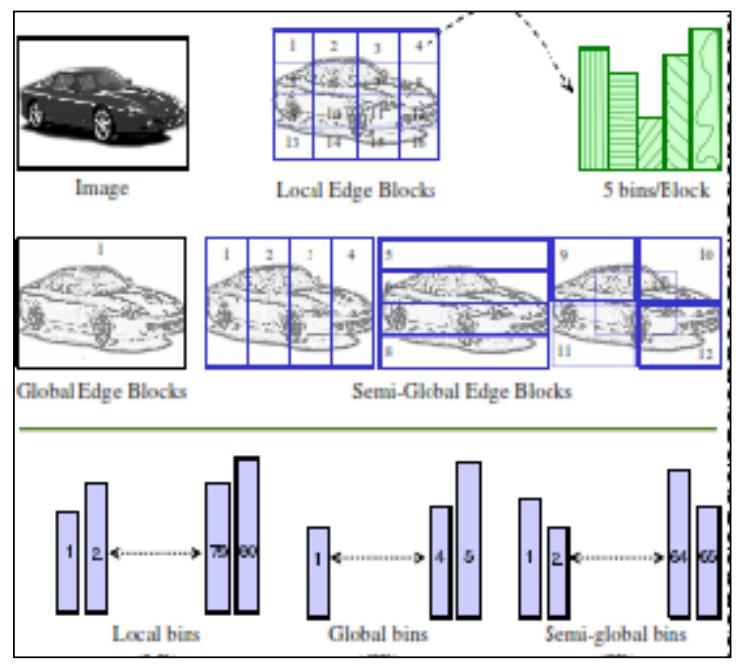

Fig 6. Edge Histogram Descriptor generation

For each block we can calculate edge distribution in 5 directions.So in this block (13*5) 65 bins are generated.The global block has 5 no of bins. The bins are generated by accumulating and normalizing the local bins.Consequently, we have a total of 150 bins ( 80 bins (local) +5 bins (global) + 65 bins (semi-global)) for the similarity matching. The similarity matching between two images $\mathrm{A}$ and $\mathrm{B}$ is calculated by the below formula.

$$
\begin{aligned}
& D(A, B)=\sum_{i=0}^{i=79} \mid \text { Local }_{-} A[i]-\text { Local }_{-} B[i] \mid \\
& +5 * \sum_{i=0}^{4} \mid \text { Global }_{-} A[i]-\text { Global }_{-} B[i] \mid \\
& +\sum_{i=0}^{64} \mid \text { Semi_Global_}_{-} A[i]-\text { Semi }_{-} \text {Global }_{-} B[i] \mid
\end{aligned}
$$

4)Similarity Matching: This involves matching these features to yield a result that is visually similar.The commonly used similarity measure method is the Distance method. There are different distances available such as Euclidean distance, City Block Distance, Canberra Distance.

5) Retrieval: The System retrieves and presents a sequence of images ranked in decreasing order of similarity or with the minimum distances is returned to the user. To evaluate the effectiveness of the proposed system precision and recall rates are to be calculated.

\section{Algoritms used by the paper:}

There are three algorithms implemented in the paper.

\section{1)Image Retrieval using Color Descriptor:}

i) Query image is given from the user.

ii) Color Descriptor Histogram of the Image is calculated. iii) Color Descriptor Histogram of the database images are calculated.

4) Euclidean Distance is calculated by the

$\mathrm{D}=\sqrt{\sum_{i=1}^{n}\left(X_{i}-Y_{i}\right)^{2}}$

5) Sorted the distance in ascending order and Top K images are displayed on the screen

\section{2)Image Retrieval using Edge Histogram Descriptor:}

i) Query image is given from the user.

ii) Edge Histogram Descriptor of the Image is calculated.

iii) Edge Histogram Descriptor of the database images are calculated.

iv) Euclidean Distance is calculated.

v) Sorted the distance in ascending order and Top K images are displayed on the screen

\section{3)Image Retrieval using Color Descriptor and Edge Histogram Descriptor:}

i) Query image is given from the user.

ii) Both features are calculated and store them in a array known as feature vector.

iii) Feature Vector of the database images are calculated.

iv) Euclidean Distance is calculated.

v) Sorted the distance in ascending order and Top K images are displayed on the screen

\section{EXPERIMENTAL EVALUATION}

A database consists of different types of images has implemented in the system. The relevant images are retrieved in the screen using different features of the color images. The result using different features are given below.Here we have also displayed how the result of csd has better than the traditional histogram method.

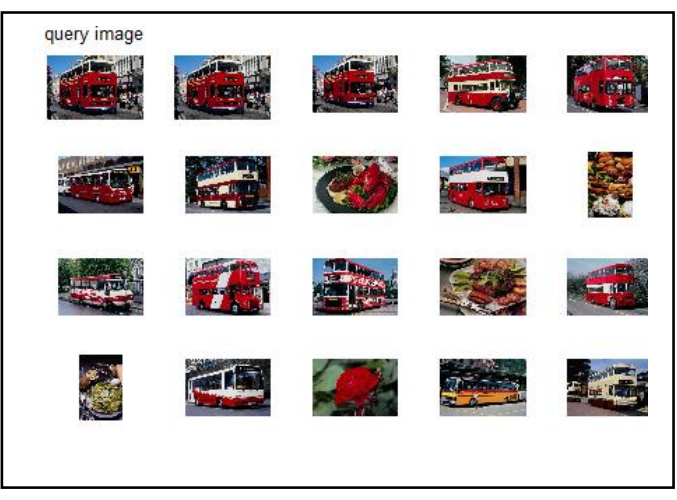

Fig 7.Output using Histogram 


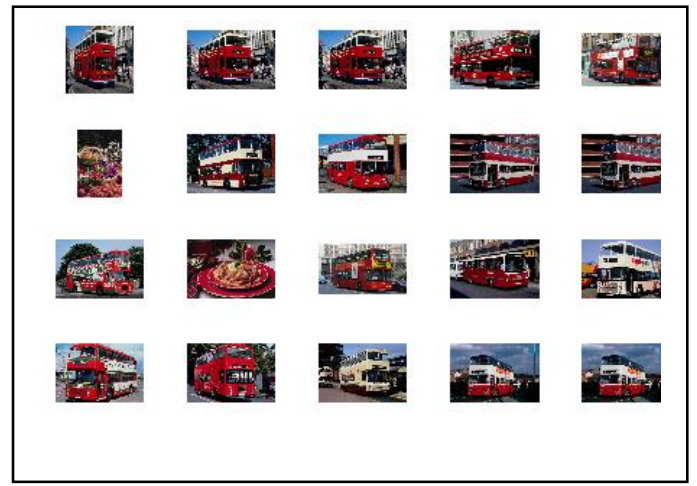

Fig 8. Output using CSD

This two diagram has clearly shown the result using CSD is better than using Histogram.

We are going to verify the result using edge histogram as feature. The result is given below.

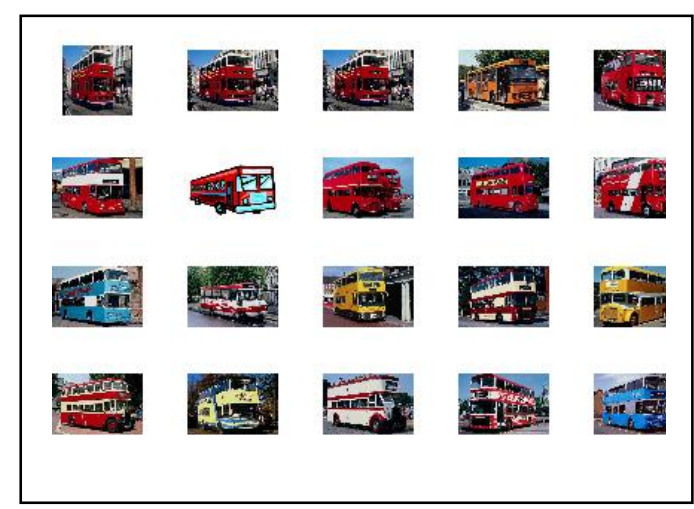

Fig 9 . Output using EHD

When we consider Edge as feature it gives better result for edge type images and during color feature the result is concentrated on color type images.So when red bus is given as the query image in fig 8 the retrievd results are more realated to color.In fig 9 for the same query image all the bus images are retrieved but the color is not proper.

Now we are going to use csd and edge histogram feature for retrieving the result.

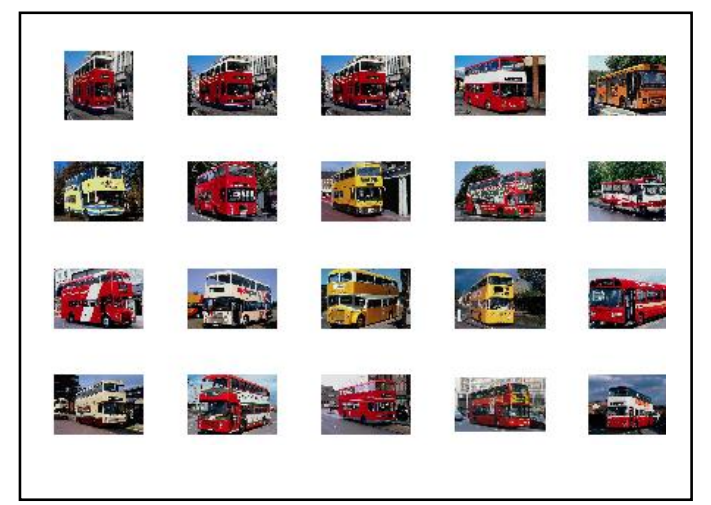

The result has clearly represented that the output using CSD and EHD has given better result than the individual feature.

We have also calculate the performance rate known as precision by using different types of images.

\section{TABLE 1 : COMPARISION OF PRECISION RATE}

\begin{tabular}{|l|l|l|l|l|l|}
\hline $\begin{array}{l}\text { Different } \\
\text { Paramete } \\
\mathbf{r}\end{array}$ & $\begin{array}{l}\text { Rose } \\
\text { imag } \\
\text { e }\end{array}$ & $\begin{array}{l}\text { Sea } \\
\text { beac } \\
\text { h }\end{array}$ & Bus & $\begin{array}{l}\text { Dinasor } \\
\text { e }\end{array}$ & $\begin{array}{l}\text { Elephan } \\
\text { t }\end{array}$ \\
\hline CSD & $89 \%$ & $53 \%$ & $\begin{array}{l}84 \\
\%\end{array}$ & $89 \%$ & $53 \%$ \\
\hline EHD & $37 \%$ & $53 \%$ & $\begin{array}{l}86 \\
\%\end{array}$ & $80 \%$ & $53 \%$ \\
\hline $\begin{array}{l}\text { CSD \& } \\
\text { EHD }\end{array}$ & $89 \%$ & $84 \%$ & $\begin{array}{l}89 \\
\%\end{array}$ & $84 \%$ & $83 \%$ \\
\hline
\end{tabular}

\section{CONCLUSION}

Efficient retrieval of images using different MPEG-7 Features is proposed in this paper. We have used a database consists of 635 images. The performance of the system is measured by using different features. The csd feature has given better result than the traditional Histogram method. We have used CSD and EHD individually in the system. Then Combinely CSD, EHD has implemented. $73 \%$ of average Precision rate is obtained by using CSD as a feature. We have got average $62 \%$ result by using Ehd. The result is improved to $85 \%$ when we have combined EHD,CSD .There is still scope of improvement in precision rate.In the present system we have not used any feedback in the output. In future we are going to used Interactive Genetic Algorithm in the relevance feedback.

\section{REFERENCES}

[1] Chih-Chin Lai, Member, IEEE, and Ying-Chuan Chen," A User-Oriented Image Retrieval System Based on Interactive Genetic Algorithm", IEEE transactions on instrumentation and measurement, vol. 60, no. 10, october 2011.

[2] Dr. D.S.Bormane Principal, RSCOE, ,Pune,India, Meenakshi Madugunki, Sonali Bhadoria, Dr. C. G. Dethe," Comparison of Different CBIR Techniques", 2011 IEEE Conference.

[3] C. Vertan, M. Zamfir, A. Drimbarean, , A. Zamfir ," MPEG-7 compliant generalized structure descriptor for still image indexing", 2011 IEEE International Conference .

[4] Wu Siqing; Xiong Gang, Wang Geng,Zhang Guoping Consumer Electronics," Digital-image retrieval based on shape-feature and MPEG-7 standard", 2011 International Conference .

[5] Jian-Hua Li, Ming-Sheng Liu, Ping Song," An novel modified extraction method of MPEG-7 visual descriptor for image retrieval”,2010 IEEE Conference.

Fig 10.Output using CSD and EHD 
[6] Hong Shao, Jun Ji, Yan Kang, Hong Zhao," Application Research of Homogeneous Texture Descriptor in Content-Based Image Retrieval”, 2009 IEEE Conference

[7] S. Kiranyaz, S. Uhlmann, M. Gabbouj," Dominant Color Extraction Based on Dynamic Clustering by Multidimensional Particle Swarm Optimization", 2009 IEEE Conference .

[8] E. Acar, S. Arslan, A. Yazici, M. Koyuncu, "Slim-tree and BitMatrix index structures in image retrieval system using MPEG-7 Descriptors",2008 IEEE Conference .
[9] Aleksey Fadeev, Hichem Frigui ," Dominant Texture Descriptors For Image Classification and Retrieval" 2008 IEEE Conference .

[10] Ka-Man Wong, Lai-Man Po, and Kwok-Wai Cheung," Dominant Color Structure Descriptor For Image Retrieval", 2007 IEEE Conference . 